were older with longer disease duration, and had higher disease activity indicators including timing of morning stiffness, 28TJC, 28SJC, PtGA, PrGA, PainVAS, ESR, CRP, DAS28-CRP, SDAl and CDAl, as well as higher radiographic assessment including $\mathrm{mTSS}$, JSN subscore and JE subscore (all $P<0.05$ ). As for $\mathrm{BMI}$ and $\mathrm{BC}$, RA patients with physical dysfunction had higher $\mathrm{BF} \%$ (mean $31.05 \%$ vs. $29.02 \%, P=0.007$ ) and prevalence of overfat $(44 \%$ vs. $27 \%, P<0.001)$, lower ASMI (mean 5.73 vs. 6.11, $P<0.001)$ and higher prevalence of myopenia $(55 \%$ vs. $38 \%, P<0.001)$ than those with normal physical function, while no difference in BMl between two groups. After adjustment confounding factors, multivariate logistic regression analysis showed overfat $(O R=2.990,95 \% C l$ : 1.695-5.274) and myopenia $(O R=1.960,95 \% \mathrm{Cl}$ : 1.191-3.225) were positively associated with physical dysfunction respectively. Further compared with patients with normal fat, patients with overfat had significantly higher rates of dysfunction of all eight physical activities including dressing, rising, eating, walking, hygiene, reaching, griping and activities respectively (all $\mathrm{P}<0.01$ ), with the highest rate $57.5 \%$ in eating dysfunction and the lowest rate $38.6 \%$ in dressing dysfunction. Comparisons between patients with and without myopenia showed similar results with the highest rate $52.4 \%$ in eating dysfunction and the lowest rate $37.7 \%$ in rising dysfunction in those with myopenia.

Conclusion: Overfat and myopenia are present in near half RA patients with physical dysfunction which are associated with dysfunction of all eight physical activities especially eating.

Acknowledgement: This work was supported by National Natural Science Foundation of China (no. 81671612 and 81801606), Guangdong Natural Science Foundation (no. 2017A030313576, 2017A030310236 and 2018A030313541) and Guangdong Medical Scientific Research Foundation (no. A2017109)

Disclosure of Interests: None declared

DOI: 10.1136/annrheumdis-2019-eular.6479

\section{SAT0082 ASSOCIATIONS OF BASELINE CLINICAL AND BIOMARKER FACTORS WITH SYMPTOMS AND FUTURE DEVELOPMENT OF CLINICALLY-APPARENT RHEUMATOID ARTHRITIS IN AN ACPA POSITIVE COHORT}

Kevin D. Deane ${ }^{1}$, Sunil Nagpal ${ }^{2}$, Navin Rao ${ }^{2}$, Frederic Baribaud ${ }^{2}$,

George Vratsanos ${ }^{2}$, Eddie A. James ${ }^{3}$, Jane H. Buckner ${ }^{3}$, Gary S. Firestein ${ }^{4}$, David L. Boyle ${ }^{4}$, Sylvia Posso ${ }^{3}$, William H. Robinson ${ }^{5}$, Laurie K. Moss ${ }^{1}$,

Saman Barzideh ${ }^{1}$, Kristen Polinski ${ }^{1}$, Sergei Ivanov ${ }^{1}$, Jennifer Seifert ${ }^{1}$, V. Michael Holers ${ }^{1} .{ }^{1}$ CU Anschutz Medical Campus, Aurora, United States of America; ${ }^{2}$ Janssen Research and Development, LLC, Spring House, United States of America; ${ }^{3}$ Benaroya Research Institute at Virginia Mason, Seattle, United States of America; ${ }^{4}$ University of California San Diego, La Jolla, United States of America; ${ }^{5}$ Stanford University, Stanford, United States of America

Background: Subjects who are in the preclinical rheumatoid arthritis (RA) state can be identified through serum elevations of circulating RA-related autoantibodies, including rheumatoid factor (RF) and antibodies to citrullinated protein antigens (ACPA), prior to the clinical appearance of inflammatory arthritis (IA) and RA. Studying Preclinical RA may identify factors and pathways in disease development, and help to identify therapeutic targets for prevention. Furthermore, trials in ACPA+ subjects have been completed or are underway with the primary goal to prevent future $|A|$ RA. However, relatively few individuals have been studied through the evolution of the Preclinical ACPA+ state to Classified RA. As such there are still many gaps in the understanding of the clinical and immunologic evolution from Preclinical to Classified RA

Objectives: The study objective is to identify the clinical, environmental and immunologic factors/pathways that are associated with incident IA/RA development in individuals with serum ACPA elevations.

Methods: We created a cohort of 86 ACPA+ subjects who at their baseline study visit did not have a historical or examination (66/68 count) evidence of IA/RA. ACPA+ was defined as a serum elevation of anti-CCP3 (IgG, Inova) on $\geq 2$ occasions above the established cut-off ( $\geq 20$ units). These subjects were recruited over 18 months through community healthfair screening, ACPA testing of first-degree relatives of patients with RA, and rheumatology clinics. Clinical, environmental and biomarker factors, including RF $\operatorname{IgA}$ and $\lg \mathrm{M}$ [Inova] and high-sensitivity C-reactive protein [hsCRP], were assessed at the baseline visit. Herein we present interim analyses of baseline visits on the whole ACPA+ cohort and longitudinal follow-up on a subset of subjects, with a specific focus on factors that are associated with baseline symptoms and subsequent incident IA/RA. Results: At baseline, the 86 ACPA+ subjects were a mean age of 58 , $66 \%$ female, $82 \%$ were Caucasian, and $40 \%$ self-reported no joint pain, morning stiffness or fatigue. hsCRP positivity ( $>3 \mathrm{mg} / \mathrm{L}$ ) was associated with increased rates of self-reported fatigue of $>0$ on VAS $(20 \%$ vs.
$41 \%, p=0.04)$. Ever smoking was associated with increased rates of selfreported morning joint stiffness (18\% vs $50 \%, \mathrm{p} \leq 0.01)$. In addition, in analyses of longitudinal follow-up data available to date, 10/86 subjects (12\%) developed IA classified as RA (2010) a median of 293 days after baseline visit. At baseline, individuals with incident RA exhibited a higher BMI compared to those who did not (27 vs. $32, p=0.03)$. In addition, positivity for hsCRP and RF, and higher median anti-CCP3 levels, were present in those who developed incident IA/RA although not statistically significant.

Conclusion: Within this new cohort of prospectively followed ACPA+ individuals, smoking and an elevated hsCRP are associated with clinical symptoms of stiffness and fatigue, respectively. The relationship of stiffness and smoking suggests this factor is related to early joint symptoms, and the association of high BMI with incident RA may indicate this is a modifiable risk factor for RA (de Hair 2013). This prospective study will continue, with further study of the factors/pathways that may influence well-being in ACPA+ individuals as well as may influence predictive of or causally linked to the future IA/RA.

Disclosure of Interests: Kevin D. Deane Grant/research support from: Janssen, Consultant for: Janssen, Sunil Nagpal Shareholder of: Johnson \& Johnson, Employee of: Janssen Research \& Development, LLC, Navin Rao Shareholder of: Johnson \& Johnson, Employee of: Janssen Research \& Development, LLC, Frederic Baribaud Shareholder of: Johnson \& Johnson, Employee of: Janssen Research \& Development, LLC George Vratsanos Shareholder of: Johnson \& Johnson, Employee of Janssen Research and Development, LLC, Eddie A. James Grant/ research support from: Janssen, Jane H. Buckner Grant/research support from: Janssen, Gary S. Firestein Grant/research support from: Janssen, David L. Boyle Grant/research support from: Janssen, Sylvia Posso Grant/research support from: Janssen, William H. Robinson Grant/research support from: Janssen, Laurie K. Moss Grant/research support from: Janssen, Saman Barzideh Grant/research support from: Janssen, Kristen Polinski Grant/research support from: Janssen, Sergei Ivanov: None declared Jennifer Seifert Grant/research support from: Janssen, V. Michael Holers Grant/research support from: Janssen, Consultant for: Janssen DOI: 10.1136/annrheumdis-2019-eular.1297

\section{SAT0083 PREVALENCE OF FRAILTY AND ITS ASSOCIATED FACTORS IN PATIENTS WITH RHEUMATOID ARTHRITIS: A CROSS-SECTIONAL ANALYSIS}

Marco DI Carlo ${ }^{1}$, Sonia Farah ${ }^{1}$, Eleonora DI Donato ${ }^{1}$, Marina Carotti ${ }^{2}$,

Fausto Salaffi'. '1 Hospital "Carlo Urbani" clinica reumatologica, Dipartimento scienze cliniche e molecolari, Jesi, Italy; ${ }^{2}$ Ospedali Riuniti Torrette Di Ancona, Dipartimento Radiologia, Torrette, Ancona, Italy

Background: The concept of frailty is a recent issue in the rheumatologic field, by now, the prevalence of frailty among individuals with rheumatoid arthritis (RA) has not been extensively examined and few studies on frailty in RA adults have been conducted. Moreover, the relationship between frailty and sociodemographic or disease characteristics in RA are unknown. Objectives: The aims of the present research were to assess the prevalence of frailty and its potential associated factors in adult patients with RA. Methods: Consecutive RA patients and healthy controls were assessed according the Survey of Health, Ageing and Retirement in Europe Frailty Instrument (SHARE-FI) (1), and classified as frail, pre-frail or non-frail. Patients underwent to a clinimetric assessment in order to establish disease activity (Simple Disease Activity Index [SDAI]), function (Health Assessment Questionnaires Disability Index [HAQ-DI]), comorbidities (modified Rheumatic Disease Comorbidity Index [mRDCl])(2), and radiographic damage (Simple Erosion Narrowing Score [SENS]). Chi-square, analysis of variance (ANOVA), and multinomial logistic regression analyses were used to test the prognostic value of frailty for the outcomes of interest.

Results: 210 consecutive RA patients were included (72 male, 138 female) and the mean age was 60.4 years. The study group was com posed by 100 healthy controls (63 females and 47 males). The mean age was 59.1. According to SHARE-FI criteria, 35 RA patients (16.6\%) were categorized as frail, $68(32.4 \%)$ as pre-frail, and $107(51 \%)$ as nonfrail, while 8 control subjects were categorized as frail, (8\%), 17 as prefrail $(17 \%)$, and 75 as non-frail $(75 \%$ ) (chi-squared $12.8 ; \mathrm{p}=0.0016$ ). The results from logistic regression analysis revealed that age (odd ratio [OR] $=1.12,95 \%$ confidence interval $[\mathrm{Cl}]=1.07-1.17 ; \mathrm{P}<0.0001)$, comorbid ities $(\mathrm{OR}=1.51,95 \% \mathrm{Cl}=1.01-2.27 ; \mathrm{P}=0.0446)$, and high disease activity $(\mathrm{OR}=1.10,95 \% \mathrm{Cl}=1.04-1.16 ; \mathrm{P}=0.0006)$ were independ ently associated with frailty in RA patients (Fig 1).

Conclusion: Frailty or pre-frailty are common in RA. The SHARE-FI may be a specific tool for the screening of frailty in RA and may summarize 